\begin{tabular}{|c|c|}
\hline Citation & $\begin{array}{l}\text { Wouter Hoogkamer, Firas Massaad, Karen Jansen, Sjoerd M. Bruijn, and } \\
\text { Jacques Duysens (2012) } \\
\text { Selective bilateral activation of leg muscles after cutaneous nerve } \\
\text { stimulation during backward walking } \\
\text { J Neurophysiol 108: 1933-1941 }\end{array}$ \\
\hline Archived version & Final publisher's version / pdf \\
\hline Published version & http://dx.doi.org/10.1152/jn.01159.2011 \\
\hline Journal homepage & http://jn.physiology.org/content/108/7/1933 \\
\hline Author contact & $\begin{array}{l}\text { wouter.hoogkamer@faber.kuleuven.be } \\
+32(0) 16329065\end{array}$ \\
\hline IR & https://lirias.kuleuven.be/handle/123456789/361893 \\
\hline & $\begin{array}{l}\text { Journal of Neurophysiology publishes original articles on the function of the } \\
\text { nervous system. It is published } 24 \text { times a year (twice monthly) by the } \\
\text { American Physiological Society, } 9650 \text { Rockville Pike, Bethesda MD 20814- } \\
\text { 3991. Copyright } \odot 2012 \text { the American Physiological Society. ESSN: 1522- } \\
\text { 1598. Visit our website at http://www.the-aps.org/. }\end{array}$ \\
\hline
\end{tabular}




\title{
Selective bilateral activation of leg muscles after cutaneous nerve stimulation
}

\section{during backward walking}

\author{
Wouter Hoogkamer, ${ }^{1}$ Firas Massaad, ${ }^{1}$ Karen Jansen, ${ }^{1}$ Sjoerd M. Bruijn, ${ }^{1,2}$ and Jacques Duysens ${ }^{1,3}$ \\ ${ }^{1}$ Motor Control Laboratory, Research Center for Movement Control and Neuroplasticity, Department of Kinesiology, \\ KU Leuven, Leuven, Belgium; ${ }^{2}$ Department of Orthopedics, First Affiliated Hospital of Fujian Medical University, Fuzhou, \\ Fujian, P.R. China; and ${ }^{3}$ Department of Research, Development, and Education, St. Maartenskliniek, Nijmegen, The Netherlands
}

Submitted 19 December 2011; accepted in final form 1 July 2012

Hoogkamer W, Massaad F, Jansen K, Bruijn SM, Duysens J. Selective bilateral activation of leg muscles after cutaneous nerve stimulation during backward walking. J Neurophysiol 108: 1933-1941, 2012. First published July 5, 2012; doi:10.1152/jn.01159.2011._During human locomotion, cutaneous reflexes have been suggested to function to preserve balance. Specifically, cutaneous reflexes in the contralateral leg's muscles (with respect to the stimulus) were suggested to play an important role in maintaining stability during locomotor tasks where stability is threatened. We used backward walking (BW) as a paradigm to induce unstable gait and analyzed the cutaneous reflex activity in both ipsilateral and contralateral lower limb muscles after stimulation of the sural nerve at different phases of the gait cycle. In BW, the tibialis anterior (TA) reflex activity in the contralateral leg was markedly higher than TA background EMG activity during its stance phase. In addition, in BW a substantial reflex suppression was observed in the ipsilateral biceps femoris during the stance-swing transition in some participants, while for medial gastrocnemius the reflex activity was equal to background activity in both legs. To test whether the pronounced crossed responses in TA could be related to instability, the responses were correlated with measures of stability (short-term maximum Lyapunov exponents and step width). These measures were higher for BW compared with forward walking, indicating that $\mathrm{BW}$ is less stable. However, there was no significant correlation between these measures and the amplitude of the crossed TA responses in BW. It is therefore proposed that these crossed responses are related to an attempt to briefly slow down (TA decelerates the center of mass in the single-stance period) in the light of unexpected perturbations, such as provided by the sural nerve stimulation.

cutaneous reflexes; human gait; phase-dependent modulation; stability; task dependency

MUSCLE REFLEX ACTIVITY plays an important role in the regulation of stable locomotion (Zehr and Duysens 2004; Zehr and Stein 1999). In humans, nonnoxious electrical stimulation of cutaneous nerves around the ankle results in reflex activity in both lower limb muscles (Duysens et al. 1990; Yang and Stein 1990) and upper limb muscles (Calancie et al. 1996; Zehr et al. 2001). This reflex activity is not just a function of background activity in the muscle, but fundamental changes in reflexes can be observed by changing features such as the stimulus intensity (Duysens et al. 1992), the stimulus location (Van Wezel et al. 1997; Zehr et al. 1997), or the task performed (Kanda and Sato 1983; Tax et al. 1995). Furthermore, within a certain task (walking, running, cycling) the reflex amplitude can change

Address for reprint requests and other correspondence: W. Hoogkamer, Motor Control Laboratory, Research Center for Movement Control and Neuroplasticity, Dept. of Kinesiology, KU Leuven, Tervuursevest 101 bus 1501, 3001 Heverlee, Leuven, Belgium (e-mail: wouter.hoogkamer@faber.kuleuven.be). between different phases of the movement cycle (Balter and Zehr 2007; Duysens et al. 1990; Mileva et al. 2004; Tax et al. 1995; Yang and Stein 1990; Zehr et al. 1997). This phasedependent modulation is typical in the reflex reversal response of the tibialis anterior (TA) muscle of the stimulated leg, where excitatory responses to tibial nerve stimulation during the early swing phase switch to suppressive responses during the late swing phase (Yang and Stein 1990). These changes were typically observed in most, but not all, participants.

It has been suggested that this phase-dependent modulation would have functional implications (Duysens et al. 1990). Indeed, Zehr and Stein (1999) reviewed the functional relevance of cutaneous reflexes during human locomotion and concluded that reflexes function "to preserve balance and ensure a stable walking pattern throughout the step cycle." More specifically, Tax et al. (1995) studied running and suggested that the reflexes in the contralateral leg (i.e., with respect to the nerve stimulation) help maintain stable locomotion, while the reflexes in the ipsilateral leg correct for the perturbation. In line with this idea, some studies pointed to important changes in the contralateral reflexes with varying degree of stability (Haridas et al. 2005, 2008; Lamont and Zehr 2007). For example, when stability was challenged by having participants walk while receiving unpredictable anterior-posterior perturbations at the waist, both with arms crossed and with freely moving arms, cutaneous reflexes in primarily the muscles of the contralateral leg were strongly affected (Haridas et al. 2005). In a follow-up study (Haridas et al. 2008) similar conditions were tested, but this time the perturbations at the waist were exchanged for mechanical perturbations at the dorsum of the ipsilateral foot. Modulated reflexes were observed in both the contralateral medial gastrocnemius (GM) and the ipsilateral TA. The contralateral leg was suggested to assist in maintaining stability while the ipsilateral leg overcomes the obstacle (Haridas et al. 2008).

Lamont and Zehr (2007) added evidence in the same direction. They collected reflex activity data from muscles in the upper and lower limbs and trunk evoked by sural nerve stimulation. Inclined walking and stair climbing were used to decrease stability and increase task uncertainty, while the effects of holding a handrail were analyzed. They observed that reflexes were amplified in muscles that were functionally able to restore balance. In line with the findings of Haridas et al. (2005, 2008), it was observed that during unsupported locomotor tasks the reflexes were most prominent in the contralateral leg muscles, which are best suited to restore balance during these tasks. Taken together, the observations in these 
studies suggest an important role for cutaneous reflexes in contralateral leg muscles in maintaining stability in locomotor tasks where stability is threatened.

Another paradigm used to induce unstable gait is backward walking (BW) (Schneider and Capaday 2003). BW is unusual and more variable than forward walking (FW) (Choi and Bastian 2007; Grasso et al. 1998; Hackney and Earhart 2009; Katsavelis et al. 2010; Kurz et al. 2012; Winter et al. 1989). Schneider and Capaday (2003) observed that the instability related to $\mathrm{BW}$ resulted in high-amplitude H-reflex activity in the soleus muscle. This reflex could be attenuated by procedures reducing the instability. In particular, they showed that when participants held the handrail or were trained in walking backward, the reflex amplitude was reduced. Subsequently, increasing instability after training (by making the participants walk backward with eyes closed) restored the large reflex activity.

Hence one would expect that BW is a good model for unstable gait and one would expect to see changes in crossed reflexes here as well. Cutaneous reflexes have been studied during BW, but only for ipsilateral reflexes (Duysens et al. 1996). It is hypothesized that the study of cutaneous reflexes in the contralateral limb during BW will provide more insight into the functional role of cutaneous reflexes, specifically in addition to earlier reports of the prominent role of contralateral cutaneous reflexes in unstable gait (Haridas et al. 2005, 2008; Lamont and Zehr 2007).

As BW is unusual and more variable than FW, we hypothesized that BW is less stable than FW and that in BW the muscles in the contralateral leg would show pronounced reflex activity during its stance phase. To test these hypotheses we applied sural nerve stimulation during BW and analyzed both the stability and the middle-latency reflex activity in both ipsilateral and contralateral lower limb muscles. To ensure that the present conditions were identical to those reported in a previous study (Duysens et al. 1996), the ipsilateral reflexes were measured as well.

\section{METHODS}

Participants and protocol. We recruited 13 healthy participants [7 men and 6 women; $26.9 \pm 3.2 \mathrm{yr}, 174.3 \pm 8.3 \mathrm{~cm}, 64.1 \pm 6.7 \mathrm{~kg}$ (mean $\pm \mathrm{SD})$ ] who had no known history of neurological or motor disorders. All participants were right-handed and right-footed. The experimental protocol was approved by the local ethics committee in accordance with the guidelines of the Declaration of Helsinki, and all participants gave written informed consent.

Participants walked both forward (FW) and backward (BW) on an instrumented dual-belt treadmill (ForceLink, Culemborg, The Netherlands), for $\sim 10 \mathrm{~min}$ per condition. For each condition we set both belt speeds to $1.1 \mathrm{~m} / \mathrm{s}(4.0 \mathrm{~km} / \mathrm{h})$, and the order of FW and BW conditions was randomized across the participants. Participants wore thin socks reaching up to $\sim 1 \mathrm{~cm}$ below the malleoli and were instructed to try to walk with the left foot on the left belt and the right foot on the right belt, but to pay little attention to any accidentally misplaced steps.

Experimental setup. We measured electromyography (EMG) recordings at 1,000 samples/s (ZeroWire, Aurion) from three muscles in both legs: the TA, GM, and biceps femoris (BF). We selected these muscles because prominent reflex activity has been observed for these muscles (Baken et al. 2006; Duysens et al. 1990, 1996). A pair of recording surface electrodes (with a diameter of $1 \mathrm{~cm}$ ) was placed over the muscle belly with an interelectrode distance of $2 \mathrm{~cm}$, parallel with the muscle fibers and close to the motor point. Care was taken not to place the electrodes over the peripheries of the muscles to minimize EMG cross talk. The electrodes were placed after hair shaving, skin abrasion, and application of alcohol and ether.

We positioned a bipolar stimulation electrode over the sural nerve, approximately halfway between the lateral malleolus and the Achilles tendon of the right leg. We determined the exact location according to the optimal irradiation of the stimulus. The electrode was then firmly attached to the skin with tape and strapped with an elastic bandage around the ankle. Each stimulus consisted of a 5-pulse (1.0 ms) train at $200 \mathrm{~Hz}$ (Grass S88 stimulator connected in series with an SIU5 isolator and a CCU1 constant-current unit, Grass Instruments). We tested both the perception and the irradiation threshold during quiet standing just before the first condition and at the end of the experiment to verify stable stimulus conditions. We set the stimulus intensity to double the perception threshold (Duysens et al. 1996).

To enable a reproducible stimulation at 16 equidistantly distributed phases in the gait cycle, we used custom-written MATLAB software that timed each stimulus in the right phase based on the instant of heel strike of the right (stimulated) leg. The instant of heel strike was determined by a vertical force (see below) threshold of $10 \%$ of body weight. Ten stimuli were presented for each of 16 phases of the gait cycle in a random order, such that there was at least one (mean 1.03) complete stride without a stimulus between consecutive stimuli. For each condition 160 stimulated strides were assessed. Before we triggered the stimulation software, subjects walked for 2-5 min on the treadmill to acclimatize to (backward) treadmill walking. The instrumented dual-belt treadmill enabled collection of the ground reaction forces for each individual leg. The forces were sampled at 1,000 samples/s. The vertical reaction force of the right (stimulated) leg was used for the online distribution of the stimuli over the gait cycle (see above). All forces were stored for off-line analyses of gait parameters.

Data analysis. We applied a fourth-order recursive, zero phaseshift, Butterworth low-pass filter with a cutoff frequency of $10 \mathrm{~Hz}$ on the raw force data. We determined step width and the instants of heel strike and toe-off based on the center of pressure (Roerdink et al. 2008). We excluded strides when a foot incidentally was placed on two belts or when two feet were on the same belt. Gait cycle was defined from right heel strike $(0 \%)$ to the next right heel strike $(100 \%)$.

To evaluate stability, we estimated the center of mass (CoM) based on the center of pressure and calculated the "extrapolated center of mass" (XCoM) and the safety margin between the XCoM and the base of support (Hof et al. 2005). Moreover, we calculated the short-term maximum Lyapunov exponent $\left(\lambda_{\mathrm{S}}\right)$ from the medio-lateral displacement of the estimated CoM, following Bruijn's protocol (Bruijn et al. 2009). We included 300 consecutive strides in the evaluation of the stability measures. Note that these strides include both stimulated and unstimulated strides.

The safety margin between the XCoM and the base of support quantifies how close an inverted pendulum model of the walking participant would be from falling. Higher values of this margin are related to more stable gait but could also result from a step-widening strategy by participants during unstable locomotion (Curtze et al. 2011). Negative values of $\lambda_{\mathrm{S}}$ indicate that the system (in this case the medio-lateral displacement of the estimated CoM) is locally stable, while positive values indicate local instability. In addition, higher values for $\lambda_{\mathrm{S}}$ imply less local stability (Bruijn et al. 2009; Dingwell and Cusumano 2000).

The EMG signals were amplified and high-pass filtered (cutoff frequency $3 \mathrm{~Hz}$ ). After full-wave rectification, we low-pass filtered the EMG signals (cutoff frequency $300 \mathrm{~Hz}$ ). We quantified the reflex responses by calculating the mean of the EMG data over the period in which the responses occurred (Fig. 1). A single 40-ms time window (Bastiaanse et al. 2006) was set around the reflexes with middle latencies of $\sim 75-80 \mathrm{~ms}$ after the stimulation (Duysens et al. 1993; Haridas et al. 2005; Yang and Stein 1990) for all 16 phases in each muscle. For each muscle, we estimated the reflex latency based on 
Stimulated stride
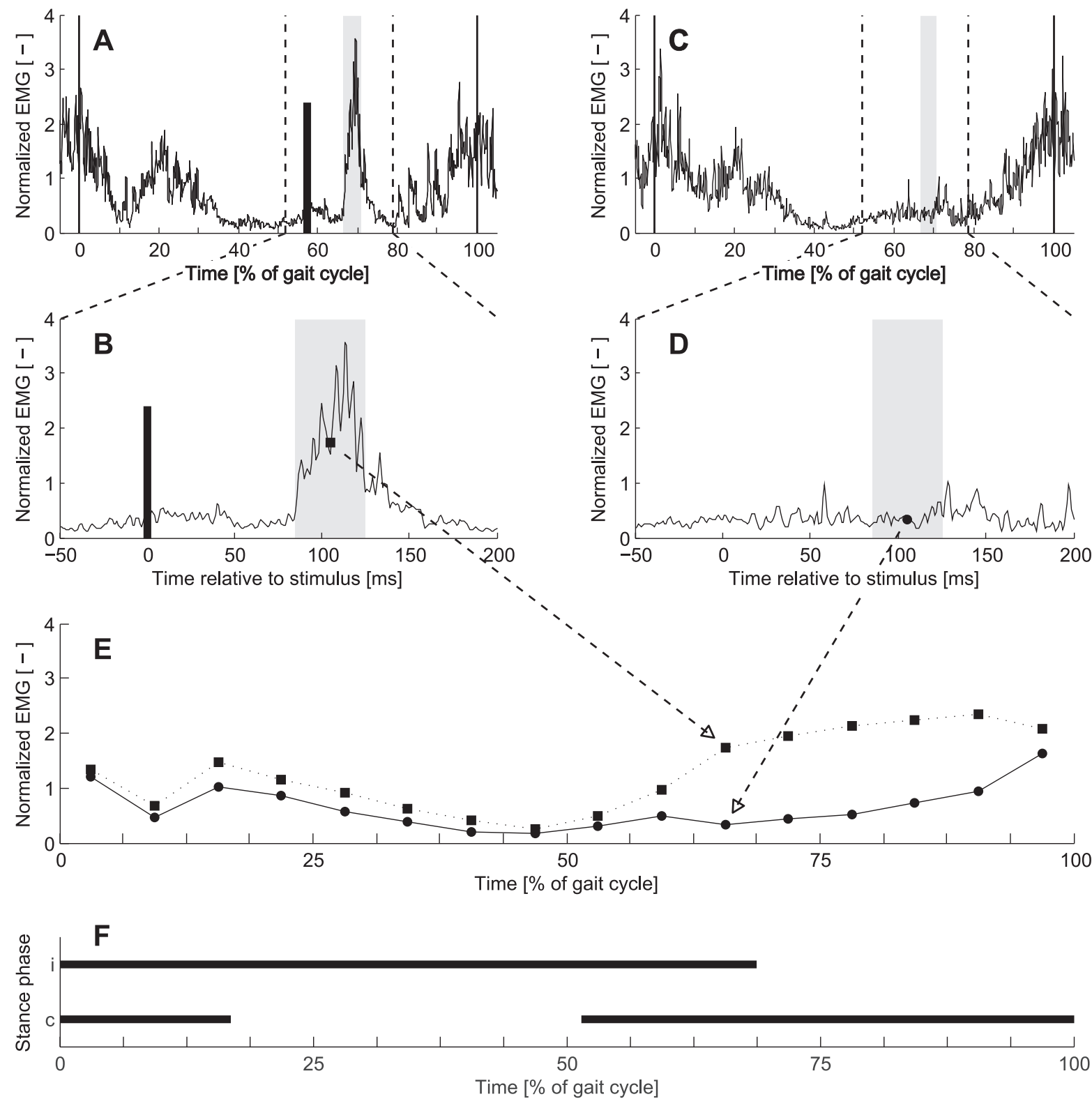

Fig. 1. A: average normalized reflex EMG of a typical stimulated stride of a single participant. Normalized EMG was averaged for 10 strides with the onset of reflex activity in the same phase of the gait cycle (in this case phase 11 of 16). The thick vertical black line indicates the instant of the nerve stimulation. $B$ : expanded view of the time frame covering the stimulus and the middle-latency response. Reflex EMG (black square) was calculated as the mean value over a 40-ms time window (gray area) starting with an individual determined latency after the stimulus (thick black line). $C$ and $D$ : background EMG (black circle) was calculated for the same relative time window of the preceding unstimulated stride. E: this procedure was repeated for all 16 phases of the gait cycle and resulted in a plot of the distribution of the reflex (squares) and background (circles) EMG over the 16 phases of the gait cycle. $F$ : duration of the stance phase for the ipsilateral (i) and contralateral (c) legs.

visual inspection of the subtracted EMG traces (see below). This latency defined the first boundary of the time window (Duysens et al. 1996). For both conditions of walking, we set the 40-ms time window at the same latency. When a muscle showed little or no response, we set the time window based on the reflex latency in other nearby muscles. For each of 16 phases of the gait cycle, we averaged the values of 10 stimulated strides. For each stimulus, we estimated the background EMG activity by calculating the mean EMG over a 40-ms time window placed at the same relative phase of the preceding unstimulated stride. Again, for each phase of the gait cycle, an average value was calculated over 10 strides. We then calculated the "net" reflex amplitude by subtracting the background EMG activity from the total reflex EMG amplitude. For intermuscle and intersubject comparison, we normalized the resulting data to the phase with the highest background EMG activity during FW.

Statistical analyses. We present all results in the text as mean values $\pm \mathrm{SD}$. We used a traditional level of significance $(\alpha=0.05)$ for all statistical tests; when appropriate, this value was corrected for the number of analyses. Paired Student's $t$-tests were applied to compare stability measures between walking directions. For the statistical analyses we log-transformed EMG values to correct for the positive skewness resulting from the full-wave rectification (see, e.g., Brown et al. 2011). To compare reflex EMG (stimulated condition) and background EMG (control condition) for all phases of the gait 
cycle we used a two-way repeated-measures ANOVA ( 2 conditions $\times$ 16 phases) for both walking directions and all muscles. For a given muscle, when we observed a significant condition main effect or a significant condition $\times$ phase interaction effect in FW or BW, we performed Tukey's honestly significant difference (HSD) post hoc analysis to determine in which phases reflex and background EMG values were significantly different.

\section{RESULTS}

During BW the participants walked with wider steps $(0.26 \pm$ $0.03 \mathrm{~m})$ than during $\mathrm{FW}(0.19 \pm 0.03 \mathrm{~m})(P<0.001)$, resulting in a higher margin of stability in $\mathrm{BW}(0.11 \pm 0.02 \mathrm{~m})$ compared with FW $(0.08 \pm 0.01 \mathrm{~m})(P<0.001)$. The $\lambda_{\mathrm{S}}$ of the medio-lateral displacement of the estimated CoM during BW was higher $(4.98 \pm 0.11)$ than during FW $(4.84 \pm 0.07)(P=$ $0.0025)$, indicating that $\mathrm{BW}$ is less stable. We had to exclude a single participant (P5) from these analyses, as one of the force sensors in the left belt was not accurately functioning.
Determination of the instants of heel strike and toe-off of the stimulated leg (right belt) was not affected, so this participant was included in the reflex analyses.

Typical results for the averaged EMG traces of the contralateral TA (cTA) muscle during backward walking for a single participant (P5) are shown in Fig. 1E. The basic observation was that there were large reflex activations in the cTA during most of contralateral stance. Similar results were obtained for the majority of participants in the population.

Population. For each phase of the gait cycle and each muscle, we calculated the average background EMG and average reflex EMG over all participants during both BW (Fig. 2) and FW (Fig. 3). To obtain the "net" reflex activity, background EMG activity was subtracted from reflex EMG activity to obtain "subtracted EMG responses" (Figs. 2 and 3, right).

In Fig. 2 it can be seen that the BW data of Fig. 1 were confirmed for most participants in the group. On the contralat-
Fig. 2. Background (circles) and reflex (squares) EMG values (left) and subtracted (reflex - background) EMG values (right) for backward walking. EMG values are averaged over all participants. Top: duration of the stance phase for the ipsilateral (i) and contralateral (c) legs. Phases when reflex EMG differs from background EMG are marked by asterisks. TA, tibialis anterior; GM, medial gastrocnemius; $\mathrm{BF}$, biceps femoris.

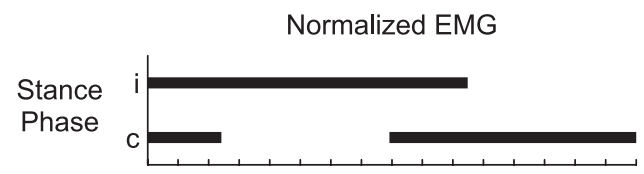

iTA

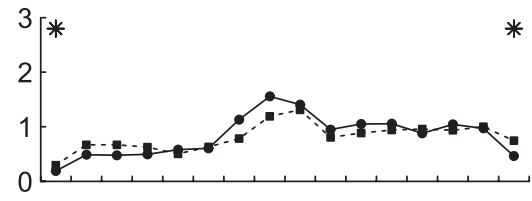

iGM

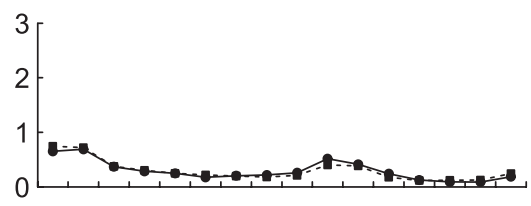

iBF

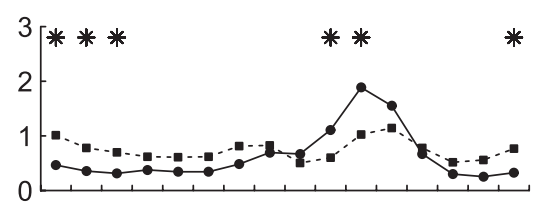

cTA
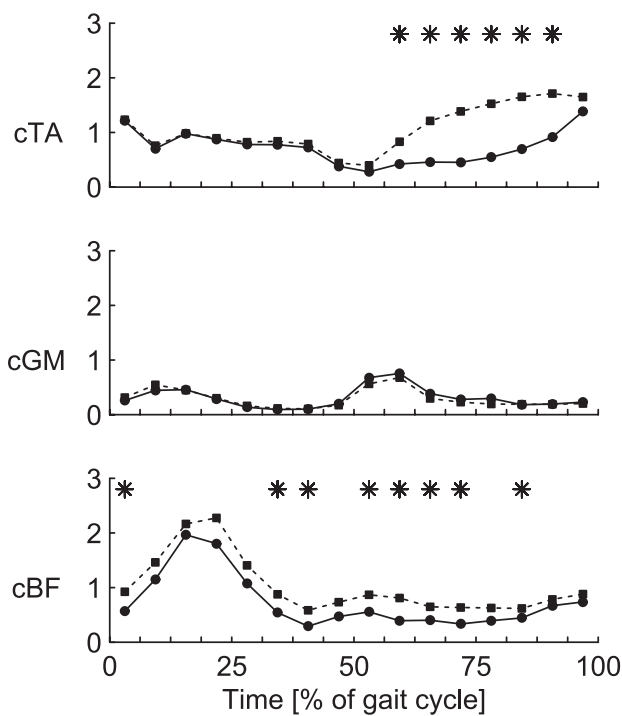

Subtracted normalized EMG
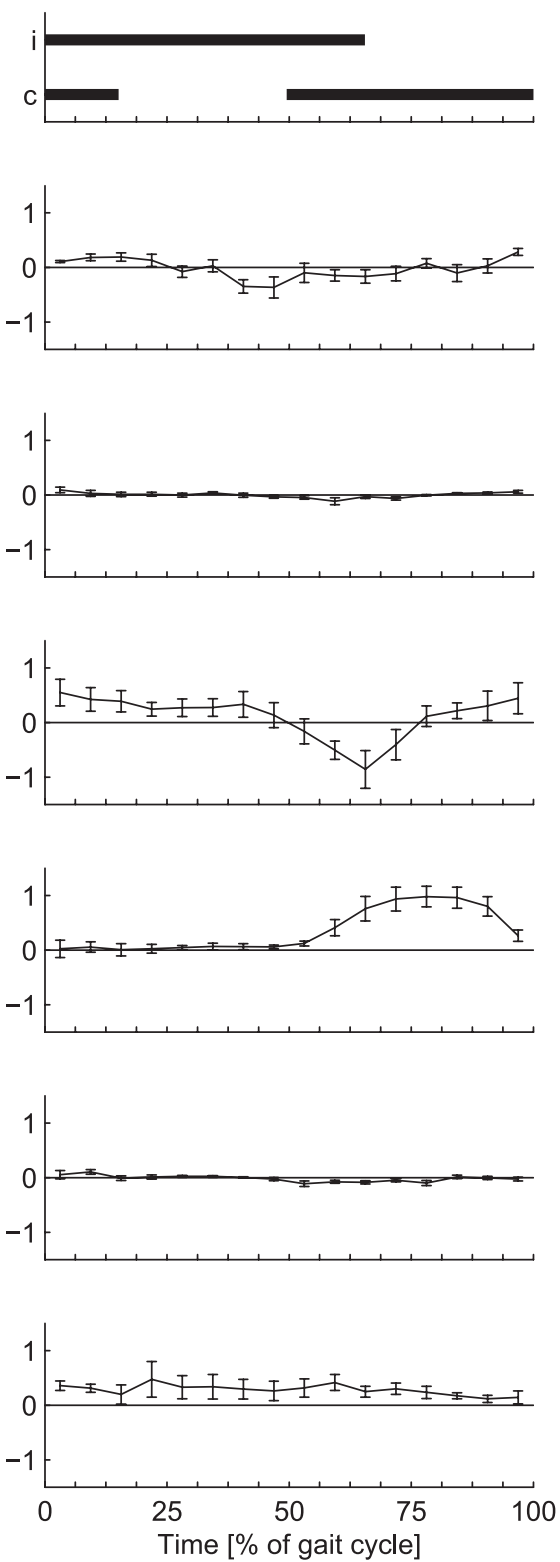
Normalized EMG
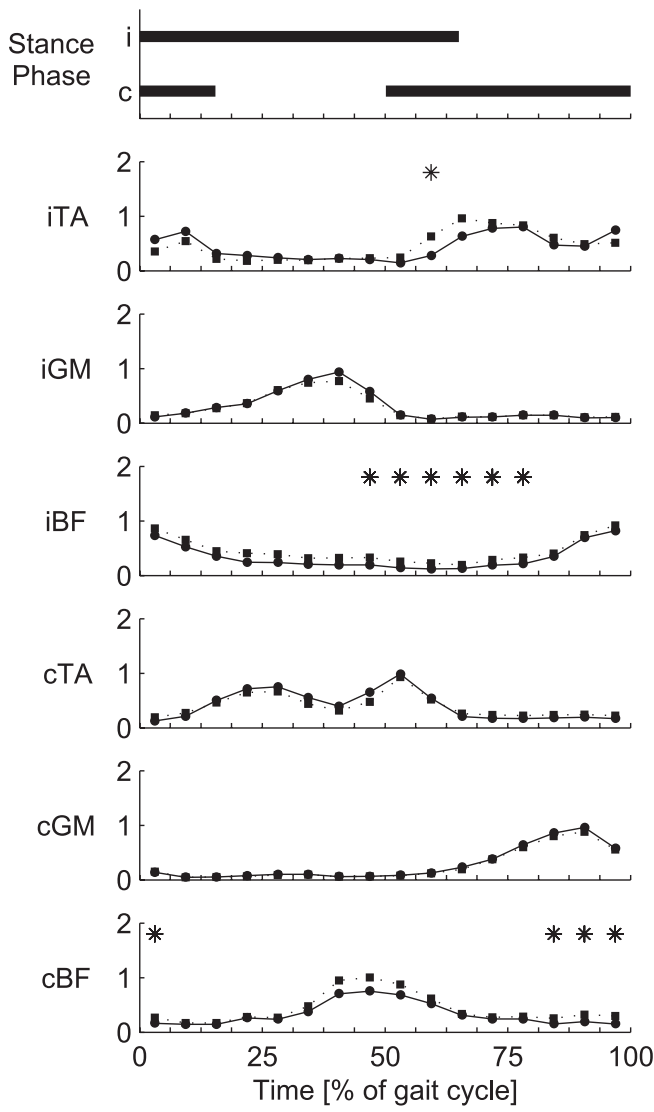

Subtracted normalized EMG
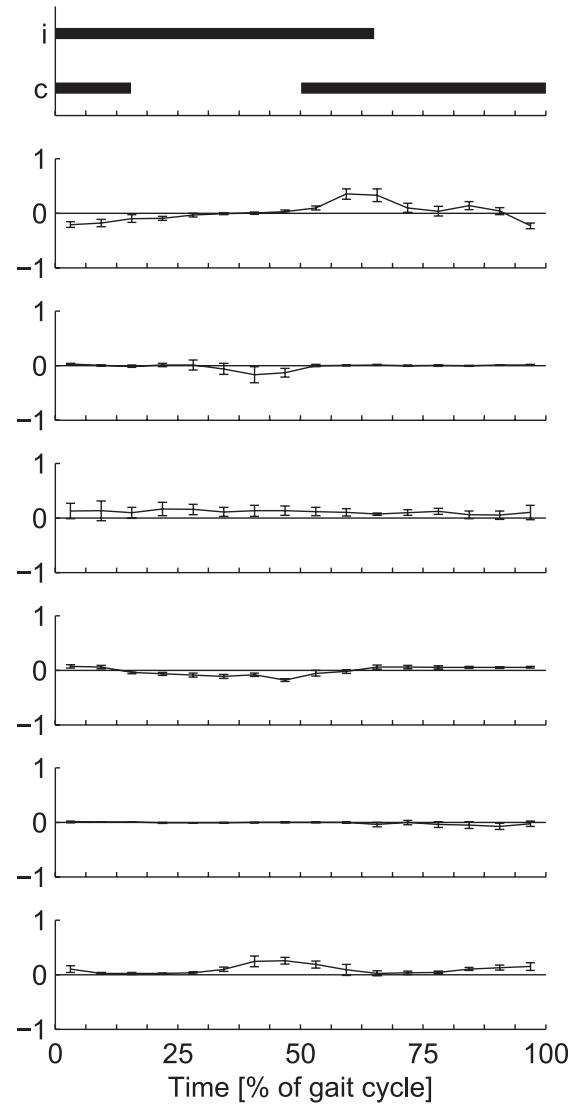

Fig. 3. Background (circles) and reflex (squares) EMG values (left) and subtracted (reflex - background) EMG values (right) for forward walking. EMG values are averaged over all participants. Top: duration of the stance phase for the ipsilateral (i) and contralateral (c) legs. Phases when reflex EMG differs from background EMG are marked by asterisks. eral side, significant reflex activity was observed in the cTA (phases 10-15) and in the BF (cBF) (phases 1, 6, 7, 9-12, and 14). At the ipsilateral side, the responses occurred in the TA (iTA) (phases 1 and 16) and BF (iBF) (phases 1-3, 10, 11, and 16). The cTA showed significantly enhanced reflex EMG activity during the middle part of the contralateral stance phase. For iBF, reflex EMG activity was below background EMG activity level (suppression) at the end of the stance phase and the start of the swing phase of the ipsilateral leg, while reflex EMG activity was enhanced during a period from the end of the swing phase to the start of the stance phase. No significant net reflex activity was observed in the ipsilateral GM (iGM) and contralateral GM (cGM): reflex EMG activity was equal to background EMG activity for all phases of the stride cycle.

For comparison, the same stimuli were also given during FW, to verify that conditions were comparable to previous studies (Baken et al. 2006; Bastiaanse et al. 2006; Duysens et al. 1992; Duysens et al. 2010; Lamont and Zehr 2007; Van Wezel et al. 1997). In FW (Fig. 3) significant reflex activity was observed in iTA (phase 10), iBF (phases 8-13), and $\mathrm{cBF}$ (phases 1 and 14-16).

Interindividual differences. As noted above, there can be substantial interindividual differences in reflex responses between participants (Baken et al. 2006). This was confirmed in the present study. Here we focus on the cTA and iBF, which displayed phase-dependent modulation on a group level (Fig. 2 ). The enhanced reflex activity in the cTA during the contralateral stance phase was observed for 10 of 13 participants (Fig. 4, right), while the background EMG activity pattern was similar for all participants.
In contrast, for the $\mathrm{iBF}$ we observed large interindividual differences both in the background EMG patterns and in the reflexes (Fig. 4, left). Several participants showed significant suppressive reflexes $(P 1-P 3, P 5)$, but for others $(P 9$ and $P 13)$ the reflex activity was enhanced in most phases of the gait cycle. These variations did not follow the variations in cTA. In other words, a tendency toward facilitation in $\mathrm{BBF}$ was not necessarily coupled to a similar tendency in TA (Fig. 4). To shed light on the basis of these variations, the reflexes were expressed in terms of a maximum gain (reflex/background) and this number was correlated with some of the available characteristics of the subjects [such as body mass, height, body mass index (BMI)] as well as with the calculated stability measures. All correlations were below $r=0.7$, and none reached significance.

\section{DISCUSSION}

In this study, we applied sural nerve stimulation during both FW and BW and analyzed the reflex activity in both ipsilateral and contralateral lower limb muscles. The most prominent new observation was the consistent presence of reflex EMG activity in the cTA during the contralateral stance phase in BW, while no significant reflex EMG activity was seen in this muscle during the swing phase, although background EMG activity was similar. In addition, we frequently observed significant reflex inhibition in the $\mathrm{iBF}$ during the transition from the ipsilateral stance phase to the ipsilateral swing phase (phases 10 and 11 ; Fig. 2). Focusing on the individual participant data of the iBF (Fig. 4), large interindividual differences in both background and reflex EMG patterns can be observed. 

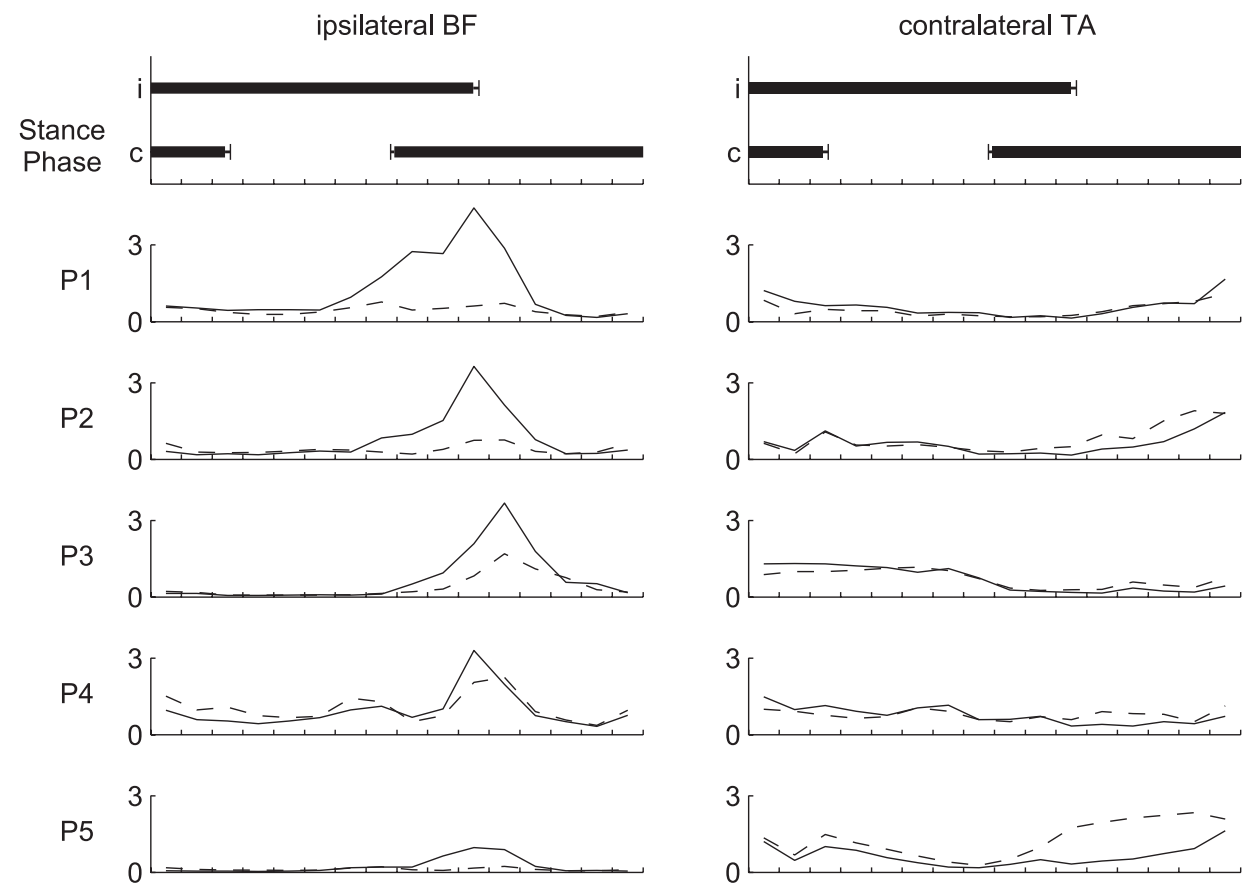

Fig. 4. Background (solid line) and reflex (dashed line) EMG values for the ipsilateral biceps femoris (left) and contralateral tibialis anterior (right) during backward walking for all individual participants. Top: duration of the stance phase for the ipsilateral (i) and contralateral (c) legs; error bars show SE.
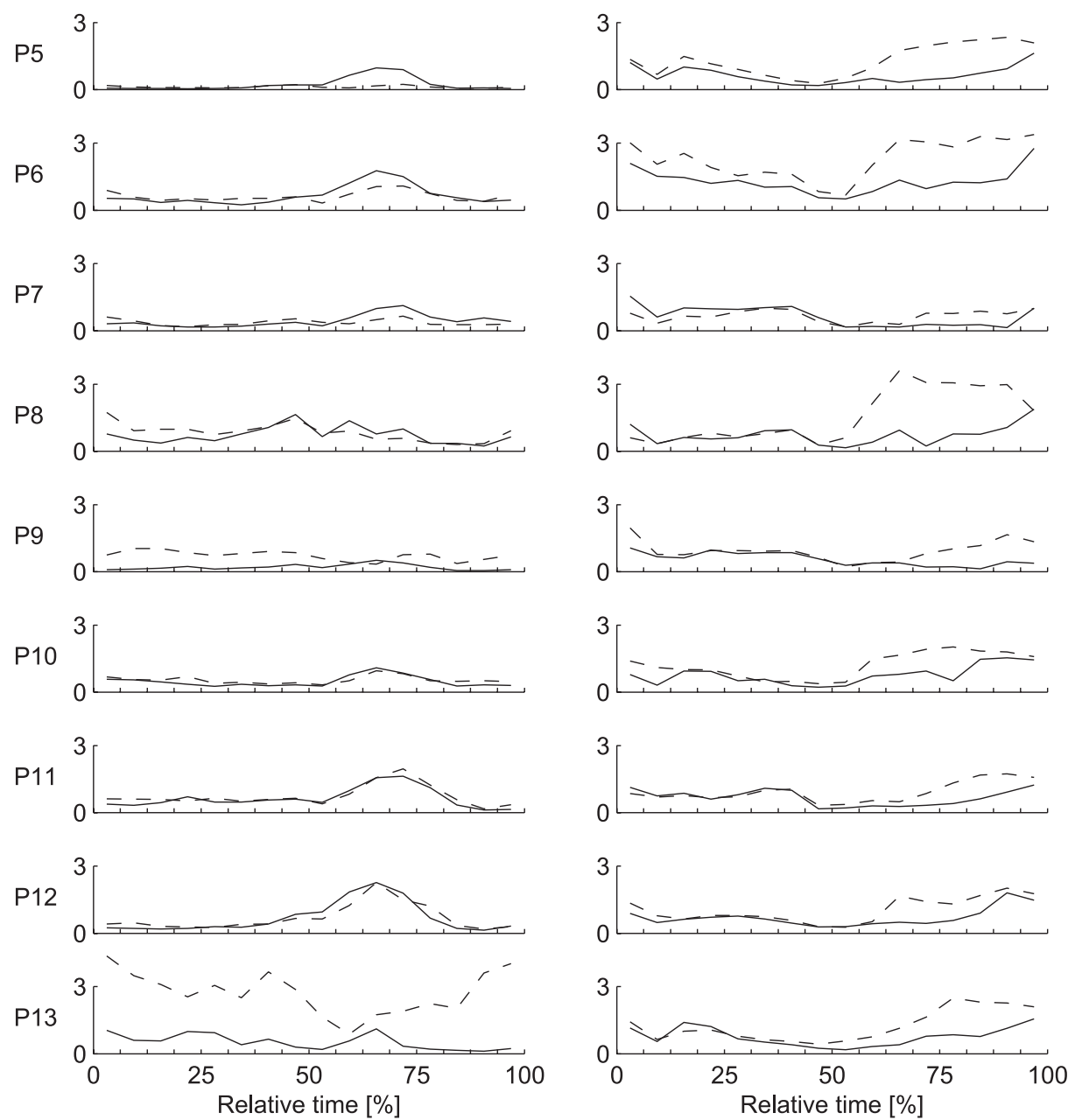

In line with earlier observations that $\mathrm{BW}$ is more variable than FW (Choi and Bastian 2007; Grasso et al. 1998; Hackney and Earhart 2009; Katsavelis et al. 2010; Kurz et al. 2012; Winter et al. 1989), we observed that BW is less locally stable than FW, i.e., $\lambda_{\mathrm{s}}$ were higher for BW. Moreover, step width and the safety margin between the XCoM and the base of support were larger for BW. The increased step width and safety margin in BW compared with FW suggests that participants increased their step width during unstable locomotion to increase the safety margin between the XCoM and their base of support (Curtze et al. 2011). Interindividual differences in the reflex activity in cTA or $\mathrm{iBF}$ (as expressed in reflex gain: reflex 
activity/background activity) were not correlated with any of the stability measures. Thus, while we observed both reduced stability and increased crossed responses in TA during BW, no correlation between these properties was observed. Therefore, no support was found for the proposal that reduced stability in BW would be the cause of pronounced reflex activity in the muscles.

The observation that the reflex activity in the cTA was mostly independent of the level of background activity suggests that during BW strong "premotoneuronal" phase-dependent modulation of reflexes occurred in this muscle. The source of this modulation is unknown, but it is striking that for crossed reflexes in BW, just as for ipsilateral reflexes in $\mathrm{FW}$, the TA was special among the muscles studied, since it consistently showed a large degree of phase-dependent modulation. It may be speculated that this is related to the large contribution of corticospinal projections in the control of this muscle during gait (Dietz 1992; Duysens et al. 2004). In addition, it is known that stimulation of the cortex induces extra TA activity during the late swing phase, before heel strike (Pijnappels et al. 1998; Schubert et al. 1997). Transcranial magnetic stimulation (TMS) is particularly effective, not only in activating TA but also in controlling sural nerve-induced reflexes (Christensen et al. 1999; Nielsen et al. 1997; Pijnappels et al. 1998). Nielsen et al. (1997) applied electrical sural nerve stimulation and TMS in seated participants during tonic voluntary contractions, and their results indicated that the TA facilitation was due to increased susceptibility of the corticomotoneuronal cells rather than an interaction between the cutaneous and corticospinal volleys at a subcortical site. In gait, similar results were obtained (Pijnappels et al. 1998). In the TA, muscular responses to paired electrical sural nerve and TMS were higher compared with the linear summation of the two stimuli separately, specifically during the swing phase of the stimulated leg. It needs to be emphasized, however, that all these data refer to the iTA and that the actual situation may be more complex for contralateral muscles.

In the $\mathrm{iBF}$, the background activity was clearly increased around the stance-swing transition of the ipsilateral leg for several participants (Pl-P4; Fig. 4). These participants also showed a large reflex inhibition during this period of the gait cycle. For other participants the background activation during the stance-swing transition was less, the accompanying reflex inhibition was less or absent, and instead reflex facilitation was observed during the first part of the ipsilateral stance phase (P8, P9, and P13; Fig. 4).

Data for cutaneous reflexes in the ipsilateral muscles during BW are available from a study by Duysens et al. (1996). The group-averaged background EMG pattern that we observed (Fig. 2) was similar to this earlier study, although we generally observed higher values over the complete gait cycle. The reflex activity pattern was also similar to the earlier study. As a result, our subtracted normalized EMG curve (Fig. 2) was similar but shifted downward, displaying more suppression. The differences between these results of both studies are most likely related to the large interindividual differences that can be observed in the iBF reflexes. Participants in our study showed varying degrees of iBF reflex inhibition, and one participant (P13) showed substantial iBF reflex facilitation, consistent over all phases of the gait cycle.
Substantial interindividual differences in reflex responses between participants have been reported previously (Bagna and Bouyer 2011; Baken et al. 2006; Duysens et al. 1992). Duysens et al. (1992) observed that most participants displayed a sural nerve TA reflex reversal, but others showed only facilitatory or only suppressive responses over the whole gait cycle. In addition, Bagna and Bouyer (2011) reported that responses to superficial peroneal and tibial nerve stimulation varied across participants. Baken et al. (2006) grouped six participants and presented the data of the seventh participant separately, as subject 7 displayed a different modulation of reflex responses compared with the remaining population. Also, large individual differences in cutaneous reflexes during gait were observed in cats (Loeb 1993). Further research focusing on interindividual differences in background and in reflex EMG is needed, to explore possible causes and relevance of these interindividual differences.

Other muscles. The responses in the contralateral muscles other than the cTA showed less dramatic phase-dependent modulation during BW. For the cGM, reflex activity was similar to the background activity for all phases of the gait cycle. The reflex modulation in the $\mathrm{cBF}$ can best be described as a continuous enhancement of the background EMG activity throughout the gait cycle.

As for the $\mathrm{iBF}$, data on the background and reflex EMG patterns of the iTA during BW are available from a study by Duysens et al. (1996). For the iTA, the presently observed reflex modulation pattern was similar, except that we observed less enhancement during the swing phase.

Generally, during FW the background and reflex EMG patterns were similar to those described in earlier studies using nonnoxious sural nerve stimulation during walking (Baken et al. 2006; Bastiaanse et al. 2006; Duysens et al. 1992, 2010; Lamont and Zehr 2007; Van Wezel et al. 1997). Increased reflex activity in the iTA muscle was repeatedly reported at the end of the stance phase and the beginning of the swing phase, followed by an inhibition at the end of the swing phase (Baken et al. 2006; Bastiaanse et al. 2006; Duysens et al. 1992, 2010; Lamont and Zehr 2007; Van Wezel et al. 1997). Reflex activity in the iBF commonly resembled the background activity pattern and was enhanced over the complete gait cycle (Baken et al. 2006; Bastiaanse et al. 2006; Duysens et al. 2010; Lamont and Zehr 2007; Van Wezel et al. 1997). Differences between our results and earlier studies could be caused by small differences in methodology. For example, several studies determined background and reflex EMG activity by averaging over a time window of individually determined length (Baken et al. 2006; Bastiaanse et al. 2006; Duysens et al. 2010), while in the present study we applied a 40-ms time window with an individually determined latency.

Less is known about reflex activity in the contralateral leg after sural nerve stimulation. Although reflexes in the cTA were not significant, the subtracted EMG patterns of the cTA and $\mathrm{cBF}$ are in agreement with earlier studies (Duysens et al. 2010; Van Wezel et al. 1997).

Functional relevance. We hypothesized that during $\mathrm{BW}$ reduced stability would cause pronounced reflex activity in the muscles of the contralateral leg. We did observe pronounced reflex activity in the CTA; however, this was not due to reduced stability. Alternatively, the pronounced reflex activity might be related to the phase-dependent function of the muscles during 
BW. In the cTA, reflex activity is most pronounced during its single-stance phase. In this period the TA resists the horizontal acceleration of the CoM (Jansen et al. 2012). To evaluate the effect of reflex activity in the TA during single stance during BW we analyzed the induced accelerations due to middlelatency responses, using a set of kinematic pilot data (A. L. Hof, unpublished observation). This analysis confirmed that reflex activity in the TA during the single-stance phase slows down the CoM.

When the sural nerve is activated during the ipsilateral swing phase, a safe strategy would be to slow down. This can be accomplished by increasing activity in the cTA, which is in agreement with the observed pronounced reflex activity. However, these activations are of short duration and were primarily seen in selected muscles such as the TA. Hence it is unlikely that they would have large behavioral consequences. This was confirmed after inspection of horizontal ground reaction force traces. There were no measurable alterations due to the sural nerve stimulation. It must be kept in mind that these responses are of small duration and therefore do not always result in large biomechanical changes. A similar situation was observed in a previous study in which auditory perturbations were applied during gait. These resulted in very minor measurable movement changes, thereby allowing for a smooth progression of gait despite clear EMG startle reactions (Nieuwenhuijzen et al. 2000). The present stimulation levels were chosen to be outside the domain of nociception. It is possible, though, that stronger stimuli would yield more dramatic changes, leading to observable decelerations of the CoM. Also, evaluation of (ankle) joint kinematics could provide further insights, similar to the study on startle reactions (Nieuwenhuijzen et al. 2000), but unfortunately no kinematic data were recorded in the present study.

Taken together, the data confirmed that BW was less stable, but no support was found for the proposal that this was the reason for the observed increased crossed responses in TA during BW. Alternatively, the pronounced reflex activity can be explained by considering the muscle's function in briefly and subtly decelerating the CoM in response to unexpected perturbations.

\section{ACKNOWLEDGMENTS}

It is a pleasure to thank At Hof for his model simulations on a set of pilot data for this project. We thank Marc Beirinckx for developing the software to control the reflex stimulation during the experiment.

\section{GRANTS}

This work was supported by KU Leuven ("bijzonder onderzoeksfonds") Grants OT/08/034 and IDO/07/012 to J. Duysens and Research FoundationFlanders (FWO) (grant G.0.756.10.N.10). F. Massaad was supported by an F+ fellowship from KU Leuven, a postdoctoral fellowship of the FWO, and a scientific prize of the Foundation Van Goethem-Brichant, Belgium.

\section{DISCLOSURES}

No conflicts of interest, financial or otherwise, are declared by the author(s).

\section{AUTHOR CONTRIBUTIONS}

Author contributions: W.H., F.M., K.J., and J.D. conception and design of research; W.H., F.M., and K.J. performed experiments; W.H., F.M., and S.M.B. analyzed data; W.H., F.M., S.M.B., and J.D. interpreted results of experiments; W.H. prepared figures; W.H. drafted manuscript; W.H., F.M.,
K.J., S.M.B., and J.D. edited and revised manuscript; W.H., F.M., K.J., S.M.B., and J.D. approved final version of manuscript.

\section{REFERENCES}

Bagna M, Bouyer LJ. A new approach for detecting and analyzing cutaneous reflexes during locomotion. J Neurophysiol 105: 1406-1415, 2011.

Baken BC, Nieuwenhuijzen PH, Bastiaanse CM, Dietz V, Duysens J. Cutaneous reflexes evoked during human walking are reduced when selfinduced. J Physiol 570: 113-124, 2006.

Balter JE, Zehr EP. Neural coupling between the arms and legs during rhythmic locomotor-like cycling movement. J Neurophysiol 97: 1809-1818, 2007.

Bastiaanse CM, Degen S, Baken BC, Dietz V, Duysens J. Suppression of cutaneous reflexes by a conditioning pulse during human walking. Exp Brain Res 172: 67-76, 2006.

Brown H, Friston K, Bestmann S. Active inference, attention, and motor preparation. Front Psychol 2: 218, 2011.

Bruijn SM, van Dieen JH, Meijer OG, Beek PJ. Statistical precision and sensitivity of measures of dynamic gait stability. J Neurosci Methods 178: 327-333, 2009.

Calancie B, Lutton S, Broton JG. Central nervous system plasticity after spinal cord injury in man: interlimb reflexes and the influence of cutaneous stimulation. Electroencephalogr Clin Neurophysiol 101: 304-315, 1996.

Choi JT, Bastian AJ. Adaptation reveals independent control networks for human walking. Nat Neurosci 10: 1055-1062, 2007.

Christensen LO, Morita H, Petersen N, Nielsen J. Evidence suggesting that a transcortical reflex pathway contributes to cutaneous reflexes in the tibialis anterior muscle during walking in man. Exp Brain Res 124: 59-68, 1999.

Curtze C, Hof AL, Postema K, Otten B. Over rough and smooth: amputee gait on an irregular surface. Gait Posture 33: 292-296, 2011.

Dietz V. Human neuronal control of automatic functional movements: interaction between central programs and afferent input. Physiol Rev 72: 33-69, 1992.

Dingwell JB, Cusumano JP. Nonlinear time series analysis of normal and pathological human walking. Chaos 10: 848-863, 2000.

Duysens J, Bastiaanse CM, Smits-Engelsman BC, Dietz V. Gait acts as a gate for reflexes from the foot. Can J Physiol Pharmacol 82: 715-722, 2004.

Duysens J, Tax AA, Murrer L, Dietz V. Backward and forward walking use different patterns of phase-dependent modulation of cutaneous reflexes in humans. J Neurophysiol 76: 301-310, 1996.

Duysens J, Tax AA, Trippel M, Dietz V. Increased amplitude of cutaneous reflexes during human running as compared to standing. Brain Res 613: 230-238, 1993.

Duysens J, Tax AA, Trippel M, Dietz V. Phase-dependent reversal of reflexly induced movements during human gait. Exp Brain Res 90: 404414, 1992.

Duysens J, Trippel M, Horstmann GA, Dietz V. Gating and reversal of reflexes in ankle muscles during human walking. Exp Brain Res 82: 351-358, 1990.

Duysens J, Van Wezel BM, Smits-Engelsman B. Modulation of cutaneous reflexes from the foot during gait in Parkinson's disease. J Neurophysiol 104: 230-238, 2010.

Grasso R, Bianchi L, Lacquaniti F. Motor patterns for human gait: backward versus forward locomotion. J Neurophysiol 80: 1868-1885, 1998.

Hackney ME, Earhart GM. Backward walking in Parkinson's disease. Mov Disord 24: 218-223, 2009.

Haridas C, Zehr EP, Misiaszek JE. Adaptation of cutaneous stumble correction when tripping is part of the locomotor environment. J Neurophysiol 99: 2789-2797, 2008.

Haridas C, Zehr EP, Misiaszek JE. Postural uncertainty leads to dynamic control of cutaneous reflexes from the foot during human walking. Brain Res 1062: 48-62, 2005.

Hof AL, Gazendam MG, Sinke WE. The condition for dynamic stability. $J$ Biomech 38: 1-8, 2005.

Jansen K, De Groote F, Massaad F, Meyns P, Duysens J, Jonkers I. Similar muscles contribute to horizontal and vertical acceleration of center of mass in forward and backward walking: implications for neural control. $\mathrm{J} \mathrm{Neu}$ rophysiol 107: 3385-3396, 2012.

Kanda K, Sato H. Reflex responses of human thigh muscles to non-noxious sural stimulation during stepping. Brain Res 288: 378-380, 1983.

Katsavelis D, Mukherjee M, Decker L, Stergiou N. Variability of lower extremity joint kinematics during backward walking in a virtual environment. Nonlinear Dynamics Psychol Life Sci 14: 165-178, 2010. 
Kurz MJ, Wilson TW, Arpin DJ. Stride-time variability and sensorimotor cortical activation during walking. Neuroimage 59: 1602-1607, 2012.

Lamont EV, Zehr EP. Earth-referenced handrail contact facilitates interlimb cutaneous reflexes during locomotion. J Neurophysiol 98: 433-442, 2007.

Loeb GE. The distal hindlimb musculature of the cat: interanimal variability of locomotor activity and cutaneous reflexes. Exp Brain Res 96: 125-140, 1993.

Mileva K, Green DA, Turner DL. Neuromuscular and biomechanical coupling in human cycling: modulation of cutaneous reflex responses to sural nerve stimulation. Exp Brain Res 158: 450-464, 2004.

Nielsen J, Petersen N, Fedirchuk B. Evidence suggesting a transcortical pathway from cutaneous foot afferents to tibialis anterior motoneurones in man. J Physiol 501: 473-484, 1997.

Nieuwenhuijzen PH, Schillings AM, Van Galen GP, Duysens J. Modulation of the startle response during human gait. J Neurophysiol 84: 65-74, 2000.

Pijnappels M, Van Wezel BM, Colombo G, Dietz V, Duysens J. Cortical facilitation of cutaneous reflexes in leg muscles during human gait. Brain Res 787: 149-153, 1998.

Roerdink M, Coolen BH, Clairbois BH, Lamoth CJ, Beek PJ. Online gait event detection using a large force platform embedded in a treadmill. $J$ Biomech 41: 2628-2632, 2008.

Schneider C, Capaday C. Progressive adaptation of the soleus H-reflex with daily training at walking backward. J Neurophysiol 89: 648-656, 2003.
Schubert M, Curt A, Jensen L, Dietz V. Corticospinal input in human gait: modulation of magnetically evoked motor responses. Exp Brain Res 115: 234-246, 1997.

Tax AA, Van Wezel BM, Dietz V. Bipedal reflex coordination to tactile stimulation of the sural nerve during human running. J Neurophysiol 73: 1947-1964, 1995.

Van Wezel BM, Ottenhoff FA, Duysens J. Dynamic control of locationspecific information in tactile cutaneous reflexes from the foot during human walking. J Neurosci 17: 3804-3814, 1997.

Winter DA, Pluck N, Yang JF. Backward walking: a simple reversal of forward walking? J Mot Behav 21: 291-305, 1989.

Yang JF, Stein RB. Phase-dependent reflex reversal in human leg muscles during walking. J Neurophysiol 63: 1109-1117, 1990.

Zehr EP, Collins DF, Chua R. Human interlimb reflexes evoked by electrical stimulation of cutaneous nerves innervating the hand and foot. Exp Brain Res 140: 495-504, 2001.

Zehr EP, Duysens J. Regulation of arm and leg movement during human locomotion. Neuroscientist 10: 347-361, 2004.

Zehr EP, Komiyama T, Stein RB. Cutaneous reflexes during human gait: electromyographic and kinematic responses to electrical stimulation. $J$ Neurophysiol 77: 3311-3325, 1997.

Zehr EP, Stein RB. What functions do reflexes serve during human locomotion? Prog Neurobiol 58: 185-205, 1999.

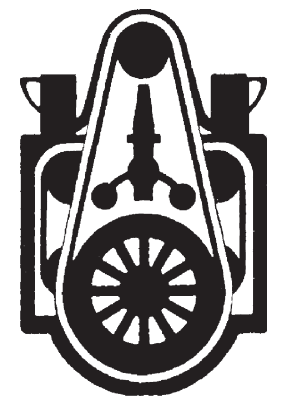

